\title{
e-Migrinter
}

17 | 2018

Enseigner les migrations internationales

\section{Enseigner les migrations internationales entre perspectives nationales et internationalisation de la formation}

David Lessault et Adelina Miranda

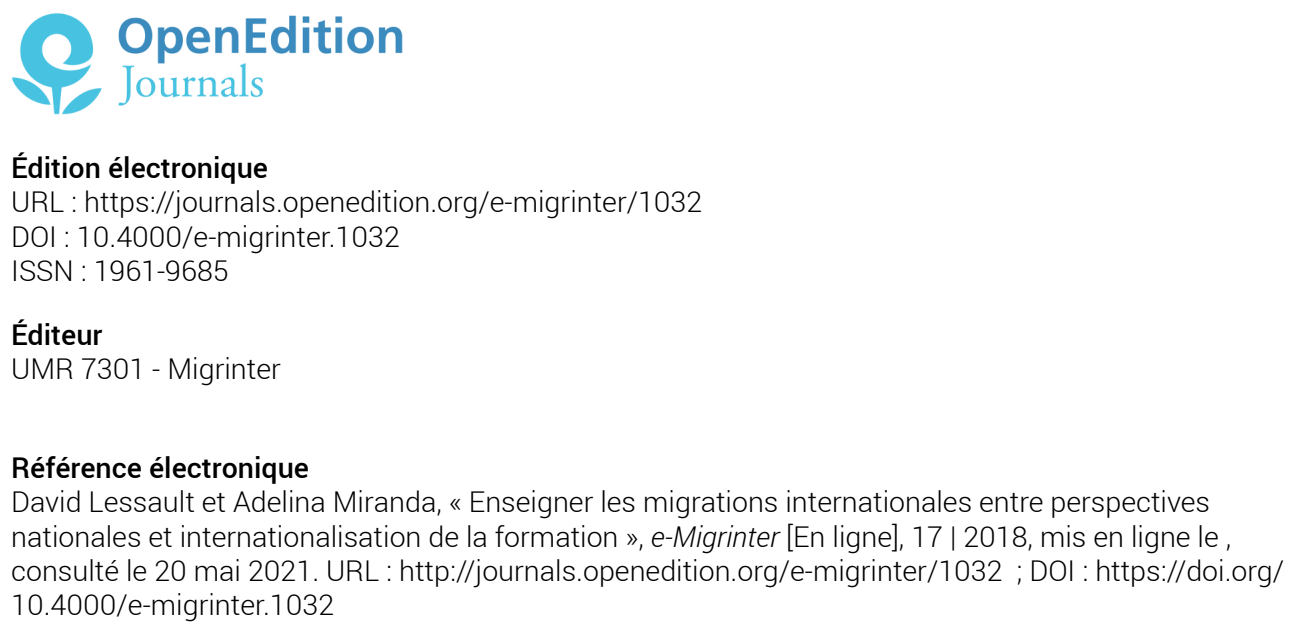

Ce document a été généré automatiquement le 20 mai 2021.

Tous droits réservés 


\title{
Enseigner les migrations internationales entre perspectives nationales et internationalisation de la formation
}

\author{
David Lessault et Adelina Miranda
}

1 À un moment où la connaissance et la compréhension des migrations internationales génèrent de fortes attentes politiques et sociales, nous souhaitons avec ce numéro porter un regard critique sur les enjeux recouverts par l'enseignement des migrations internationales. Ce dossier approfondit cette question à partir de l'étude de six pays (Argentine, Belgique, France, Italie, Mexique et Sénégal) et montre la complexité à traiter une question qui croise tant les perspectives nationales que l'internationalisation de la formation. Les contributions présentées permettent de constater que les études des mouvements des populations traversent deux échelles de la production des savoirs : d'un côté, elles relèvent d'une pratique pédagogique ancrée dans le nationalisme méthodologique et, de l'autre côté, elles se situent dans la circulation de paradigmes, théories et idées au niveau international.

2 L'enseignement des migrations, en tant qu'analyseur et révélateur d'une vision nationaliste des sociétés et des cultures, traduit le récit de l'histoire des États-Nations. La manière dont les programmes scolaires et universitaires proposent l'étude des migrations traduit la place que les États-Nations confèrent aux étrangers et aux nationaux. Pendant longtemps les analyses se sont limitées à regarder l'arrivée des étrangers dans les pays d'accueil en terme d'insertion. Avec l'accélération des échanges internationaux et une prise de conscience que les enjeux économiques et politiques nationaux se jouent aussi à l'échelle mondiale, la manière d'enseigner les migrations en simples termes d'intégration a atteint ses limites.

3 Un bilan de trente ans de recherches en sciences sociales sur les migrations permet de constater que le discours et les débats promus par les recherches scientifiques portent désormais sur le «transnationalisme », l'intensification des « circulations migratoires » 
et le paradigme mobilitaire. L'une des avancées des études migratoires est donc d'avoir ajouté une certaine complexité dans la description, la mesure et la compréhension des faits migratoires dont les ressorts évoluent rapidement et demandent une mise en relation intelligible avec les changements des espaces de départ, d'arrivée et de transit. Toutefois, il n'est pas évident qu'en dehors des laboratoires et des formations spécialisées l'idée que les migrations soient pluri-directionnelles, réversibles et de plus en plus complexes car situées dans des temporalités multiples et des espaces à géométrie variable ait gagné l'ensemble des mondes académique, politique et professionnelle. Autrement dit, que les principales avancées scientifiques en la matière soient parvenues, par diffusion et transmission des savoirs, aux différentes sphères de la société.

4 En même temps, nous devons constater que les migrations internationales peuvent être utilisées comme un formidable outil pédagogique pour le programme d'histoiregéographie qui se décline de plus en plus, comme on peut le lire dans les manuels scolaires, au prisme de la «mondialisation ». Faire du parcours détaillé d'un migrant, un prétexte utile à l'étude des réalités économiques et sociales de plusieurs pays (ceux du départ, de la « traversée » et d'arrivée), de la nature des relations entre les États, des enjeux personnels et familiaux qui se fabriquent dans le projet migratoire, n'est-ce pas enseigner une géographie ouverte sur les mondes et l'altérité ?

Une autre avancée récente dans les études migratoires est relative à la production de cadres théorique et méthodologique qui ont permis la mise au point d'un apparat conceptuel qui circule entre les différentes disciplines des sciences sociales. Face à la démultiplication des offres de formation sur les migrations, il est cependant important de situer les contextes de production des savoirs. L'histoire de Migrinter (G. Simon), de l'Urmis (S. Potot) ou du Colegio de la Frontera Norte (C. Pizzonia) montre une adéquation de la transmission des connaissances sur les migrations aux contextes institutionnels. Il en ressort l'idée forte que les objectifs des enseignements - à tous les niveaux - se jouent dans l'interdisciplinarité. L'importance de construire un cadre pédagogique pluridisciplinaire est mis en avant par tous les auteurs qui contribuent à ce numéro ; toutefois, selon l'appartenance des uns et des autres, les angles d'analyse divergent. Les diverses "sensibilités disciplinaires" (sociologique, géographique, démographique, économique, anthropologique) sont productrices de grilles de lecture spécifiques qui reposent sur une diversité d'approches et de méthodes.

6 Dès lors, trois grandes questions se posent. Face à la diversité croissante de disciplines enseignées et/ou d'appartenance des enseignants-chercheurs, existe-t-il des concepts transversaux, voire un/des paradigme(s) « support » en vue d'enseigner les migrations internationales dans une perspective pluridisciplinaire? Les échanges générés par la visée d'un projet commun de formation axé sur les migrations et la mobilité des hommes et des femmes à l'échelle internationale peuvent-ils permettre de dépasser les principaux paradigmes «mobilitaire », « développementaliste » et "transnational »? La transmission du langage universitaire à celui de l'enseignement secondaire est-elle vraiment assurée et assumée?

7 Ces questions renvoient à la nécessité de réfléchir sur les modalités de circulation des savoirs. Les conditions qui président à la mobilité des chercheurs, grâce à l'internationalisation de la formation et de l'enseignement (notamment à travers Erasmus, les cotutelles et les contrats post-doctoraux), favorise les échanges. Toutefois, la formation continue à être traversée par une sorte de domination scolaire qui 
s'enracine dans la perspective occidental-centrée des savoirs. M. B. Timéra, I. F. Diouf, M. Diongue et P. Sakho soulignent qu'au Sénégal les formations empruntent les structures des discours dominants et que les enseignements ne tiennent pas compte des sensibilités différentes entre Sud et Nord. Il est donc important de rappeler l'utilité tant d'un changement des cadres de pensée sur les migrations internationales que de la mise en place d'une action politique plus large. Enseigner les migrations internationales d'une manière critique passe tout d'abord par une remise en cause des paradigmes et des méthodes utilisés. Cette condition constitue la base pour œuvrer pour une reconnaissance des continuités existant entre situation postcoloniale et migrations (S. Potot) et du fait que les migrants et leurs descendants constituent l'une composante non négligeable des sociétés européennes (N. Ouali).

8 La prise en compte des hiérarchies qui traversent la construction des savoirs et des méthodologies pour étudier les migrations permettrait une convergence des regards scientifiques mais également politiques entre les « Nords» et les "Suds». Elle serait certainement un excellent moyen de prendre de la distance avec les discours à courte vue qui prolifèrent dans certains médias et débats politiques et qui ont souvent pour effet de dérouter l'opinion publique.

Que l'on enseigne ou se forme en France, au Sénégal ou bien en Argentine, l'effort consiste d'abord à tenir compte des différents points de vue et d'observation des migrations comme nous l'explique en ouverture du dossier Gildas Simon dans des propos recueillis et organisés par Véronique Lassailly-Jacob. Constat est ainsi fait que les différents contextes institutionnels, historiques et politiques se reflètent dans l'enseignement des migrations et que les formations semblent être ancrées dans les représentations «nationales" ou "régionales » du phénomène. Des contributions portant sur son enseignement en Belgique (Nouria Ouali), en Italie (Fabio Amato, Rosa Gatti), au Mexique (Cristina Pizzonia) et en Argentine (Susanna Masseroni), il ressort une forte imbrication entre les contextes de production scientifique, les évolutions épistémologiques (construction du savoir sur les migrations) et les pratiques pédagogiques mises en œuvre (manière de transmettre les connaissances sur les migrations). De plus, la perspective disciplinaire ou le regard scientifique adoptés semblent varier selon les lieux ou les contextes à partir desquels les migrations sont enseignées. C'est ce qui ressort aussi bien du texte sur l'enseignement des migrations dans les collèges et lycées du Sénégal (Mamadou Bouna Timéra, Ibrahima Faye Diouf, Momar Diongue, Papa Sakho) que de l'expérience pédagogique qui est analysée par Christelle Maridet et Antoine Dumont, à travers la mise en place d'un atelier pédagogique auprès d'élèves de 4ème dans un collège français situé en milieu rural.

Pour la première fois, ce numéro d'E Migrinter publie dans son dossier des textes courts et également en langues étrangères. C'est l'expression d'une double volonté : celle d'ouvrir l'espace de publication à un public de professionnels, notamment enseignants, souhaitant partager leurs réflexions et expériences pédagogiques sur les migrations ; celle de poursuivre la démarche d'ouverture internationale qui alimente depuis ses origines le projet scientifique du laboratoire Migrinter. 


\section{AUTEURS}

\section{DAVID LESSAULT}

Chargé de recherche au CNRS, Migrinter - UMR 7301 CNRS / Université de Poitiers david.lessault@univ-poitiers.fr

\section{ADELINA MIRANDA}

Professeure d'anthropologie à l'Université de Poitiers, directrice de Migrinter, UMR 7301 CNRS / Université de Poitiers

adelina.miranda@univ-poitiers.fr 